\title{
Long-term outcomes of internet-delivered cognitive behaviour therapy for paediatric anxiety disorders: towards a stepped care model of health care delivery
}

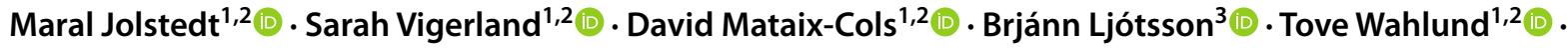 \\ Martina Nord $^{1,2}$ (D) . Jens Högström ${ }^{1,2}$ (D) Lars-Göran Öst $^{4}$ (D) Eva Serlachius ${ }^{1,2}$ (D)
}

Received: 2 October 2019 / Accepted: 12 September 2020 / Published online: 22 September 2020

(C) The Author(s) 2020

\begin{abstract}
Internet-delivered cognitive behaviour therapy (ICBT) is emerging as a powerful tool to fill the gap between demand and availability of evidence-based treatment for paediatric anxiety disorders. However, it is still unclear how to best implement it in routine clinical care. 123 children (8-12 years) with anxiety disorders underwent a 12-week ICBT programme with limited therapist support. Participants were assessed 3- and 12-month post-ICBT (3MFU and 12MFU, respectively). Non-remitters who still fulfilled diagnostic criteria for their principal anxiety disorder at 3MFU were offered additional manualised "faceto-face" (F2F) CBT. The aim of the study was to emulate a stepped-care model of health care delivery, where the long-term treatment gains of ICBT as well as the potential benefit of proving addition treatment to non-remitters of ICBT were evaluated. Remitters of ICBT ( $n=73$ ) continued to improve throughout the study period (pre-ICBT to 12MFU; Cohen's $d=2.42$ ). At $12 \mathrm{MFU}, 89 \%(n=65)$ were free from their principal anxiety disorder. Of all the participants classed as non-remitters at 3MFU $(n=37), 48.6 \%(n=18)$ accepted the offer to receive additional F2F CBT. These participants also improved with a large effect from pre-ICBT to $12 \mathrm{MFU}$ (Cohen's $d=2.27$ ), with the largest effect occurring during F2F CBT. At 12MFU, $83 \%(n=15)$ were free from their principal anxiety disorders. The majority of non-remitters declining additional F2F CBT $(63.2 \% ; n=12)$ did so due to already receiving treatment at their local CAMHS, prior to 3MFU. The effects of ICBT for anxiety disorders are durable at least up to 1 year after the end of treatment. Patients who fail to fully benefit from ICBT improved further with additional F2F sessions at our clinic, suggesting that it may be feasible to implement ICBT within a stepped-care model of health care delivery.
\end{abstract}

Keywords Child psychiatry $\cdot$ Anxiety disorders $\cdot$ Cognitive behaviour therapy $\cdot$ Health services accessibility $\cdot$ Telemedicine

Electronic supplementary material The online version of this article (https://doi.org/10.1007/s00787-020-01645-x) contains supplementary material, which is available to authorized users.

Maral Jolstedt

maral.jolstedt@ki.se

1 Child and Adolescent Psychiatry Research Centre, Department of Clinical Neuroscience, Karolinska Institutet, Gävlegatan 22, 11330 Stockholm, Sweden

2 Stockholm Health Care Services, Stockholm County Council, Stockholm, Sweden

3 Division of Psychology, Department of Clinical Neuroscience, Karolinska Institutet, Stockholm, Sweden

4 Department of Psychology, Stockholm University, Stockholm, Sweden

\section{Introduction}

Paediatric anxiety disorders are common [1] and often accompanied by a range of related problems if left untreated [2]. Development of early, accessible and cost-efficient interventions is therefore imperative. Cognitive behaviour therapy (CBT) is an efficacious first line treatment for anxiety disorders in children [3] but access to it is limited [4]. Remote delivery of CBT via the Internet (ICBT) is both efficacious and cost-effective for young people with anxiety disorders [5, 6] and has the potential to greatly increase the availability of treatment [7]. However, the long-term effects of ICBT for paediatric anxiety disorders have rarely been studied. To date, only three trials have investigated whether the treatment gains of ICBT for paediatric anxiety disorders were maintained at long-term follow-up [8-10]. All three 
studies suggested that treatment gains were not only maintained but there were indications of further improvement at follow-up. However, these studies had substantial data loss at follow-up and none recorded additional treatments received during the follow-up period, casting some doubt about the long-term effects of ICBT.

Assuming that the effects of ICBT for anxiety disorders are durable, it is still unclear how to best implement this novel treatment modality in routine clinical care. ICBT is often quoted as a potential early intervention in the context of a stepped-care model [11], where patients are first offered a low-intensity intervention, reserving higher intensity treatments to more complex cases or to those who fail to benefit sufficiently [12]. Though stepped-care approaches may intuitively seem like an ideal model for psychiatric service delivery, evidence for their feasibility in real-world settings is scarce [12-15].

This study reports the naturalistic 1-year follow-up data from participants in a large randomised controlled trial (RCT) of ICBT for paediatric anxiety disorders [6]. As part of the study protocol, all participants $(N=131)$ received ICBT either immediately post-randomisation or after crossover from the control intervention. Emulating a stepped-care mode of healthcare delivery, participants who required additional treatment at 3-months follow-up (3MFU), were systematically offered face-to-face CBT (F2F CBT) at our clinic. Additional treatments were carefully recorded throughout the follow-up period for all patients. Our specific research questions were: (1) For patients who were in remission at $3 \mathrm{MFU}$, were their treatment gains maintained up to 12 months after treatment completion? (2) For patients classed as non-remitters at $3 \mathrm{MFU}$, was additional F2F CBT associated with improvements in symptoms and functioning?

\section{Methodology}

\section{Participants}

Participants with a principal diagnosis of separation anxiety disorder, generalised anxiety disorder, specific phobia, social anxiety disorder or panic disorder were recruited through newspaper advertisements and referrals from the child- and adolescent mental health service (CAMHS) or primary care centres in Sweden. The study inclusion- and exclusion criteria are listed in Supplementary Table 1. Caregivers provided written consent and children provided verbal assent prior to participating in the study. The study protocol was approved by the Stockholm Regional Ethical Review Board (reference numbers 2014/1885-31 and 2015/316-31/1) and the trial was registered at ClinicalTrials.gov (number NCT02350257).

\section{Study design}

For full details of the study design, please see [6]. Briefly, participants were randomly allocated to receive either 12 weeks of ICBT $(n=66)$ or of an active control condition called Internet-delivered child directed play $(n=65)$. Participants allocated to the control condition were offered to cross over to ICBT immediately after the primary endpoint (week 12), regardless of whether they were in remission. Fifty-seven participants, of whom six were classed as being in remission, accepted the offer and started a course of ICBT. The remaining eight dropped-out from the study during or directly after the control condition and did not provide further data. In total, a pooled sample of $N=123$ participants received ICBT (i.e., 66 immediately and 57 crossing over). Of these 123 participants, 117 still met diagnostic criteria for their principal anxiety disorder at the time they started ICBT (Supplementary Fig. 1).

Participants were recruited to the original RCT during March 11, 2015-October 21, 2016. The period of followup lasted from 1 October, 2015 to April 2, 2018; see Fig. 1 for a flow chart and assessment points throughout the present trial. Participants still meeting diagnostic criteria for their principal anxiety disorder at $3 \mathrm{MFU}$ were offered to receive additional $\mathrm{F} 2 \mathrm{~F}$ treatment at our clinic.

\section{Measures}

The primary outcome measure was the Clinician Severity Rating (CSR) of the principal anxiety disorder derived from the Anxiety Disorder Interview Schedule for DSMIV: parent and child versions (ADIS-C/P) [16]. In ADIS, the severity of each diagnosis is rated with the CSR on a 9-point Likert scale, ranging from 0 ("Absence of symptoms/No disturbance in functioning/No disability") to 8 ("Very severe symptoms/Very severe disturbance in functioning/Very severely disabling"). A rating of four or higher indicates that the child meets diagnostic criteria for the disorder. The full range (0-8) was assigned in the current trial. The ADIS C/P has shown good to excellent test-retest reliability and inter-rater reliability [17] as well as concurrent validity [18]. The inter-rater reliability in this trial based on the principal anxiety disorder was good ( $\mathrm{ICC}=0.77 ; 95 \% \mathrm{CI}=0.49-0.89$ ) for $\mathrm{CSR}$ and fair $(\kappa=0.57,95 \%$ CI $0.38-0.76)$ for assessing diagnostic status.

Secondary outcome included diagnostic status assessed with the ADIS; measures of clinician assessed functional impairment with the Children's Global Assessment Scale (CGAS) [19]; self and parent reported anxiety symptoms with the Revised Children's Anxiety and Depression Scale 


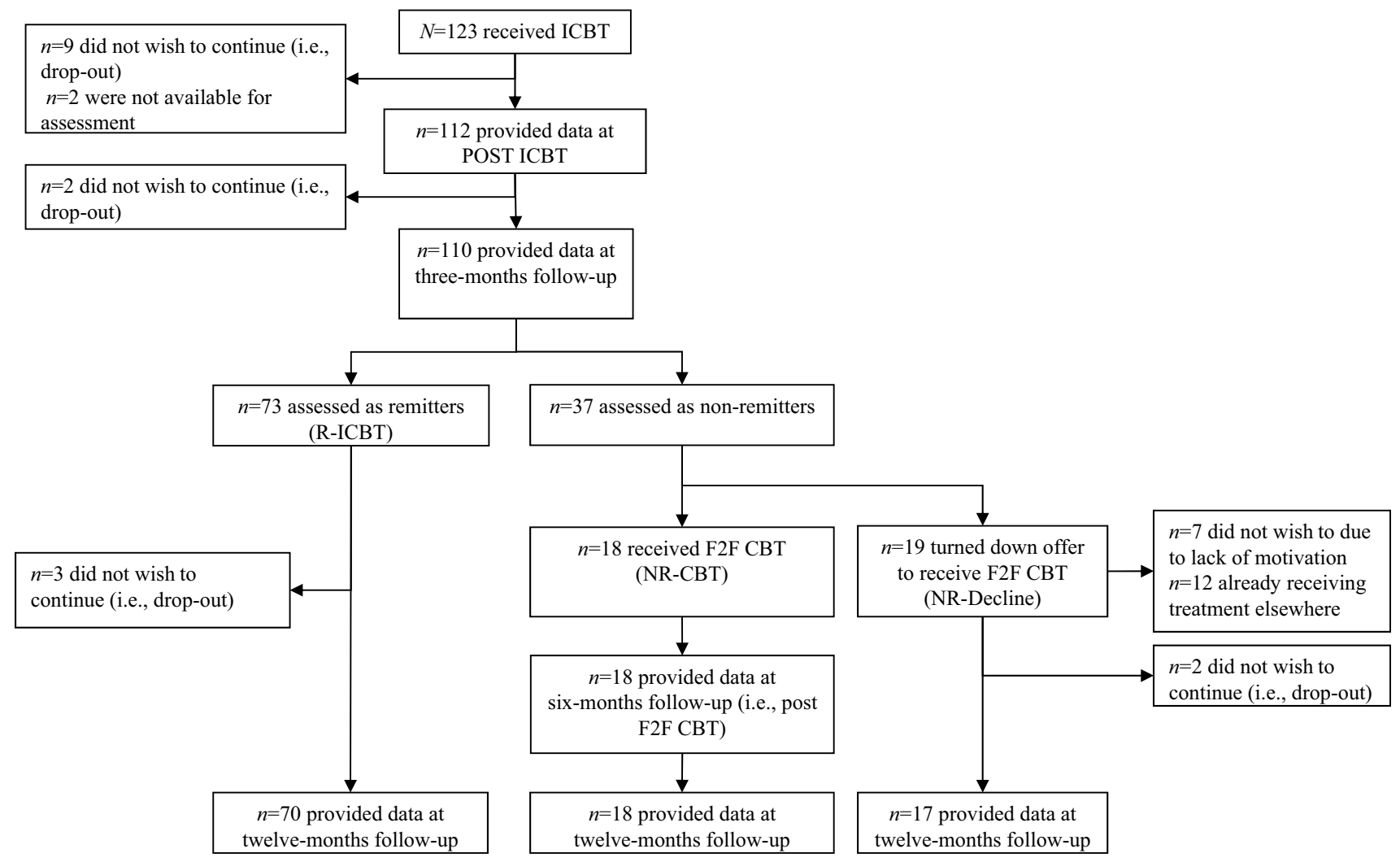

Fig. 1 Participant flow through-out the trial. ICBT Internet-delivered Cognitive Behaviour Therapy; F2F CBT Face-To-Face Cognitive Behaviour Therapy; $R-I C B T$ Remitters of Internet-delivered Cognitive Behaviour Therapy; $N R$-CBT Non-remitters of Internet-delivered Cognitive Behaviour Therapy receiving additional face-to-face Cog- nitive Behaviour Therapy; NR-Decline Non-remitters of Internetdelivered Cognitive Behaviour Therapy declining the offer to receive additional face-to-face Cognitive Behaviour Therapy. Participants already receiving treatment elsewhere started treatment prior to 3-months follow-up assessment
(RCADS-C/P) [20, 21]; self and parent reported functional impairment with the Work and Social Adjustment Scale (WSAS-C/P) [22]; as well as self and parent reported quality of life with the KIDSCREEN-10 [23]. The RCADS$\mathrm{C} / \mathrm{P}$ consists of two subscales, the major depression subscale and the total anxiety subscale. The latter was used in the current trial. Parental anxiety and depression were measured with the Hospital Anxiety and Depression Scale (HADS) [24]. We also carefully documented whether the child had received additional treatment elsewhere, defined as either CBT or medication with SSRI including change in dosage during the follow-up period; and whether any adverse events were experienced during ICBT or F2F CBT; see Supplementary Table 2 and 3 for an overview of the measures used and when during the study period they were administered.

\section{Interventions}

\section{Internet-delivered CBT}

The ICBT protocol (BiP Anxiety) is a completely webbased, therapist-guided self-help CBT programme for children with anxiety disorders and their caregivers. The treatment contains 12 consecutive modules for the child to complete together with one or both parents and 12 separate modules directed to the parent only. The duration of the treatment is 12 weeks and one module needs to be completed before gaining access to the next. The intervention primarily consists of self-guided exposure therapy with asynchronous and personalised therapist support provided on a weekly basis, via messages in the treatment platform and worksheets within the programme. For a summary 
of the content of the BiP Anxiety programme; see Supplementary Table 4 .

\section{Face-to-face CBT for non-remitters}

According to the study protocol, all participants classed as non-remitters (still meeting diagnostic criteria for their principal anxiety disorder) at the 3MFU were offered additional manualised F2F CBT at the Stockholm Child and Adolescent Psychiatry Research Centre, a specialised research unit within CAMHS in Stockholm. The F2F CBT manual had the same treatment content as the ICBT programme, with emphasis on exposure, but was delivered in a traditional face-to-face format. Therapists used a structured manual with instructions as well as worksheets that could be used throughout the treatment. Participants were offered up to 10 sessions of F2F CBT ( $60 \mathrm{~min} / \mathrm{session})$ during 12 weeks and treatment included both the child and at least one parent. For all participants, the first session started with a joint discussion about the possible reasons why ICBT had not had the desired effect, followed by collaborative treatment planning (e.g., which components to include, how many sessions at each phase, type and amount of parental involvement). The last session mainly focused on maintenance of treatment gains and relapse prevention strategies. Disorder specific components, such as imaginal exposure for generalised anxiety disorder, social skills training for social anxiety disorder and interoceptive exposure for panic disorder, as well as cognitive techniques could be administered when needed. For details on specific treatment components that were delivered; see Supplementary Table 5.

\section{Analysis}

\section{Research question 1}

For participants in remission at $3 \mathrm{MFU}$ (R-ICBT; $n=73$ ), a piecewise linear mixed model (LMM) [25] was fitted to determine within-group symptom change over time on the different continuous variables. LMM models use all available data and are ideally suited to handle missing data. The model included two knots to detect and specify the change that occurred between different time points, specifically from (1) pre- to post-ICBT, (2) post-ICBT to 3MFU and (3) 3MFU-12MFU. We also ran an additional LMM including the receipt of any additional treatments during the follow-up period (coded yes/no) as a covariate.

\section{Research question 2}

Because not all patients classed as non-remitters accepted the offer of additional F2F CBT post hoc analyses were conducted on this sub-sample. Two separate LMM models were used. For those who received F2F CBT (NR-CBT; $n=18$ ), the LMM included an additional knot/spline (i.e., total of three knots) to specify the change from 3 to $6 \mathrm{MFU}$ (i.e., from pre- to post-F2F CBT). For participants declining the offer to receive additional CBT, despite still fulfilling criteria for their principal anxiety disorder (NR-Decline; $n=19$ ), the LMM was fitted with only two knots.

\section{Linear mixed models}

In an additional post hoc analysis, three separate LMM were fitted without any knots, to determine the total change from pre-treatment to $12 \mathrm{MFU}$ for each of the three groups (R-ICBT, NR-CBT and NR-Decline). Effect sizes (Cohen's d) for all changes, between the different time points, were calculated with the estimates derived from the LMM together with the pooled observed standard deviation [26]. When available, measures reported during treatment were included in all the conducted LMM. Further, all LMM were built by starting with a fixed intercept and fixed effect of time and then sequentially adding (1) a random intercept and/or (2) a random effect of time. The final model for each fitted LMM was determined by using the chi-square goodness-of-fit test.

\section{Other considerations}

In this study, remission was defined as no longer fulfilling diagnostic criteria at a given assessment point (i.e., CSR rating $<4$ ) and is presented for both principal anxiety disorder as well as all anxiety disorders. Univariate ANOVA with post hoc Bonferroni multiple comparisons (continuous measures) and Kruskal-Wallis test with post hoc Mann-Whitney $U$ test with Bonferroni adjusted alpha value (categorical measures) were used when comparing three groups (R-ICBT, NR-CBT and NR-Decline). Participants with the missing data were compared with those who did provide data at $12 \mathrm{MFU}$ (yes/no) by conducting independent samples $t$ tests on the whole sample $(N=123)$. Assumptions for parametric tests were tested by using normal probability plots, residual plots and Cook's distance. Statistical significance was set at $p<0.05$ and 95\% CI was used. All statistical analyses were conducted in SPSS version 25 .

\section{Results}

\section{Clinical characteristics}

The demographic and clinical characteristics of the total sample and the various subgroups according to remission status are summarised in Table 1. Pre-treatment 
Table 1 Demographic Features and Clinical Characteristics at pre-treatment

\begin{tabular}{|c|c|c|c|c|c|c|c|}
\hline & Total sample $N=123$ & R-ICBT $n=73$ & NR-CBT $n=18$ & NR-Decline $n=19$ & $F$ value & Kruskal-Wallis & $p$ value \\
\hline Female, $n(\%)$ & $65(52.8)$ & $37(50.7)$ & $13(72.2)$ & $9(47.4)$ & & 3.03 & 0.220 \\
\hline Age, $M(S D)$ & $9.93(1.35)$ & $9.89(1.37)$ & $9.94(1.47)$ & $9.89(1.20)$ & 0.01 & & 0.998 \\
\hline $\begin{array}{l}\text { Previous contact with } \\
\text { CAMHS, } n(\%)\end{array}$ & $61(49.6)$ & $33(45.2)$ & $9(50.0)$ & $11(57.9)$ & & 0.99 & 0.609 \\
\hline Ongoing medication, $n(\%)$ & $12(9.8)$ & $7(9.6)$ & $1(5.6)$ & $3(15.8)$ & & 1.11 & 0.575 \\
\hline \multicolumn{8}{|l|}{ Principal anxiety disorder, $n(\%)$} \\
\hline SEP & $48(39.0)$ & $34(46.6)$ & $5(27.8)$ & $4(21.1)$ & & 5.23 & 0.073 \\
\hline SAD & $25(20.3)$ & $11(15.1)$ & $5(27.8)$ & $8(42.1)$ & & 6.85 & 0.033 \\
\hline GAD & $23(18.7)$ & $11(15.1)$ & $3(16.7)$ & $5(26.3)$ & & 1.33 & 0.515 \\
\hline SP & $20(16.3)$ & $12(16.4)$ & $4(22.2)$ & $2(10.5)$ & & 0.92 & 0.632 \\
\hline PD & $7(5.7)$ & $5(6.8)$ & $1(5.6)$ & $0(0)$ & & 1.36 & 0.507 \\
\hline Number of diagnoses, $M(S D)$ & $1.81(1.18)$ & $1.60(0.83)$ & $1.67(0.69)$ & $2.58(1.84)$ & 6.38 & & 0.002 \\
\hline $\begin{array}{l}\text { CSR score, principal anxiety } \\
\text { disorder, } M(S D)\end{array}$ & $4.56(0.81)$ & $4.41(0.85)$ & $4.72(0.83)$ & $5.00(0.67)$ & 4.33 & & 0.016 \\
\hline $\begin{array}{l}\text { Depressive symptoms, } M \\
(S D)\end{array}$ & $6.85(4.22)$ & $6.46(4.08)$ & $6.33(5.02)$ & $7.83(4.36)$ & 0.95 & & 0.391 \\
\hline $\begin{array}{l}\text { Parental anxiety and depres- } \\
\text { sion, } M(S D)\end{array}$ & $8.87(6.84)$ & $8.60(6.22)$ & $8.28(7.43)$ & $8.74(6.66)$ & 0.03 & & 0.975 \\
\hline \multicolumn{8}{|l|}{ Educational level, parent, $n(\%)$} \\
\hline$<12$ years & $7(5.7)$ & $4(5.5)$ & $0(0)$ & $1(5.3)$ & & 1.02 & 0.601 \\
\hline 12 years & $16(13.0)$ & $9(12.3)$ & $1(5.6)$ & $3(15.8)$ & & 0.97 & 0.614 \\
\hline University studies & $18(14.6)$ & $10(13.7)$ & $2(11.1)$ & $5(26.3)$ & & 2.13 & 0.345 \\
\hline Graduate degree & $79(64.2)$ & $48(65.8)$ & $14(77.8)$ & $10(52.6)$ & & 2.57 & 0.277 \\
\hline $\mathrm{PhD}$ & $3(2.4)$ & $2(2.7)$ & $1(5.6)$ & $0(0)$ & & 1.07 & 0.587 \\
\hline Age, parent, $M(S D)$ & $42.67(4.72)$ & $42.62(4.52)$ & $43.22(5.04)$ & $41.95(5.94)$ & 0.32 & & 0.728 \\
\hline
\end{tabular}

$R$-ICBT Remitters of Internet-delivered Cognitive Behaviour Therapy; NR-CBT Non-remitters of Internet-delivered Cognitive Behaviour Therapy receiving additional face-to-face cognitive behaviour therapy; NR-Decline Non-remitters of Internet-delivered Cognitive Behaviour Therapy declining the offer to receive additional face-to-face cognitive behaviour therapy; CAMHS child and adolescent mental health services; SEP separation anxiety disorder; $G A D$ generalized anxiety disorder; $S A D$ social anxiety disorder; $S P$ specific phobia; $P D$ panic disorder; $C S R$ Clinician Severity Rating derived from the Anxiety Disorder Interview Schedule

Parental anxiety and depression symptoms measured with the Hospital Anxiety and Depression Scale; Depressive symptoms self-reported with the depressive symptoms subscale of The Revised Children's Anxiety and Depression Scale

comparisons showed that participants in the NR-Decline group included more children with social anxiety disorder as their principal diagnosis, when compared with participants in the R-ICBT group $(z=2.58 ; p=0.010)$. Participants in the NR-Decline group also had higher clinician severity rating (CSR) of the principal anxiety disorder as compared to participants in R-ICBT (mean difference $=0.59 ; p=0.018$ ) and a higher number of anxiety diagnoses compared to both participants in R-ICBT (mean difference $=0.96 ; p=0.002$ ) and NR-CBT (mean difference $=0.91 ; p=0.030$ ). No other significant differences at pre-treatment were observed. Severity of the anxiety disor$\operatorname{der}(t=0.15, p=0.882)$, degree of functional impairment $(t=1.00, p=0.320)$ and number of comorbid diagnoses $(t=1.38, p=0.171)$ did not differ significantly when comparing those with the missing data at $12 \mathrm{MFU}$ as compared to those who provided data.

\section{Long-term outcomes for remitters after ICBT}

ICBT-treated remitters $(n=73)$ continued to improve on the primary outcome measure (CSR of the principal anxiety disorder), with a large effect size from post-treatment to $3 \mathrm{MFU}(d=0.88 ; 95 \% \mathrm{CI}=0.62-1.15)$ and a further small effect size from 3 to $12 \mathrm{MFU}(d=0.42 ; 95 \%$ $\mathrm{CI}=0.17-0.68)$. Considering the entire trial period, from pre-treatment to $12 \mathrm{MFU}$, remitters improved with a large effect size $(d=2.42 ; 95 \% \mathrm{CI}=1.78,3.07)$; see Table 2 for a full summary of primary and secondary outcomes from pre-treatment to $12 \mathrm{MFU}$ in this group.

Seven participants $(10.3 \%)$ relapsed from being in remission at $3 \mathrm{MFU}$ to meeting criteria for their principal anxiety disorder at $12 \mathrm{MFU}$. Thus, a total of 61 participants $(89.7 \%)$ were still in remission at $12 \mathrm{MFU}$. Further, 53 participants $(77.9 \%)$ were free from all anxiety disorders at $12 \mathrm{MFU}$. 
Table 2 Primary- and secondary outcome measures for remitters of ICBT $(n=73)$

\begin{tabular}{|c|c|c|c|c|c|c|}
\hline & \multicolumn{3}{|c|}{ Observed values, per protocol } & \multicolumn{3}{|c|}{ Estimated change, intent-to-treat } \\
\hline & Time & $n$ & $M(S D)$ & Time & Cohen's $d(95 \% \mathrm{CI})$ & $p$ value \\
\hline \multirow[t]{4}{*}{ CSR } & Pre & 73 & $4.41(0.85)$ & Pre-12MFU & $2.42(1.78,3.07)$ & $<0.001$ \\
\hline & Post & 73 & $2.93(0.89)$ & Pre-Post & $1.71(1.27,2.15)$ & $<0.001$ \\
\hline & $3 \mathrm{MFU}$ & 73 & $2.15(0.88)$ & Post-3MFU & $0.88(0.62,1.15)$ & $<0.001$ \\
\hline & $12 \mathrm{MFU}$ & 70 & $1.73(1.08)$ & $3 \mathrm{MFU}-12 \mathrm{MFU}$ & $0.42(0.17,0.68)$ & $<0.001$ \\
\hline \multirow[t]{4}{*}{ CGAS } & Pre & 73 & $58.88(6.79)$ & Pre-12MFU & $1.07(0.76,1.37)$ & $<0.001$ \\
\hline & Post & 72 & $66.71(8.52)$ & Pre-Post & $0.99(0.74,1.24)$ & $<0.001$ \\
\hline & $3 \mathrm{MFU}$ & 70 & $69.67(9.36)$ & Post-3MFU & $0.34(0.14,0.55)$ & 0.001 \\
\hline & $12 \mathrm{MFU}$ & 69 & 70.58 (10.75) & $3 \mathrm{MFU}-12 \mathrm{MFU}$ & $0.08(-0.09,0.25)$ & 0.376 \\
\hline \multirow[t]{4}{*}{ RCADS-C } & Pre & 71 & $30.06(15.48)$ & Pre-12MFU & $1.14(0.77,1.52)$ & $<0.001$ \\
\hline & Post & 68 & $22.16(13.83)$ & Pre-Post & $0.54(0.33,0.75)$ & $<0.001$ \\
\hline & 3MFU & 61 & $18.80(12.13)$ & Post-3MFU & $0.28(0.07,0.49)$ & $<0.001$ \\
\hline & $12 \mathrm{MFU}$ & 52 & $14.62(11.85)$ & 3MFU-12MFU & $0.34(0.10,0.58)$ & 0.001 \\
\hline \multirow[t]{4}{*}{ RCADS-P } & Pre & 73 & 33.37 (12.08) & Pre-12MFU & $1.22(0.86,1.58)$ & $<0.001$ \\
\hline & Post & 73 & $22.44(11.61)$ & Pre-Post & $0.98(0.74,1.22)$ & $<0.001$ \\
\hline & $3 \mathrm{MFU}$ & 66 & 20.89 (12.28) & Post-3MFU & $0.11(-0.11,0.33)$ & 0.192 \\
\hline & $12 \mathrm{MFU}$ & 59 & $17.31(11.88)$ & $3 \mathrm{MFU}-12 \mathrm{MFU}$ & $0.28(0.06,0.49)$ & 0.003 \\
\hline \multirow[t]{4}{*}{ WSAS-C } & Pre & 71 & $11.18(7.23)$ & Pre-12MFU & $1.07(0.67,1.48)$ & $<0.001$ \\
\hline & Post & 68 & $7.56(6.10)$ & Pre-Post & $0.55(0.28,0.82)$ & $<0.001$ \\
\hline & $3 \mathrm{MFU}$ & 61 & $6.23(5.63)$ & Post-3MFU & $0.30(0.06,0.53)$ & 0.019 \\
\hline & $12 \mathrm{MFU}$ & 52 & $4.12(5.38)$ & $3 \mathrm{MFU}-12 \mathrm{MFU}$ & $0.34(0.06,0.61)$ & 0.024 \\
\hline \multirow[t]{4}{*}{ WSAS-P } & Pre & 73 & $15.82(7.46)$ & Pre-12MFU & $1.45(1.03,1.87)$ & $<0.001$ \\
\hline & Post & 73 & $9.95(7.33)$ & Pre-Post & $0.79(0.52,1.07)$ & $<0.001$ \\
\hline & $3 \mathrm{MFU}$ & 66 & $7.55(5.41)$ & Post-3MFU & $0.33(0.14,0.53)$ & 0.002 \\
\hline & $12 \mathrm{MFU}$ & 59 & $5.15(4.94)$ & $3 \mathrm{MFU}-12 \mathrm{MFU}$ & $0.42(0.16,0.68)$ & 0.005 \\
\hline \multirow[t]{4}{*}{ KIDSCREEN-C } & Pre & 70 & $40.36(4.65)$ & Pre-12MFU & $0.41(0.11,0.71)$ & 0.004 \\
\hline & Post & 68 & $40.34(5.10)$ & Pre-Post & $0.00(-0.21,0.21)$ & 0.990 \\
\hline & $3 \mathrm{MFU}$ & 61 & $40.44(5.69)$ & Post-3MFU & $0.01(-0.18,0.21)$ & 0.894 \\
\hline & $12 \mathrm{MFU}$ & 52 & $42.63(4.78)$ & 3MFU-12MFU & $0.34(0.04,0.65)$ & 0.003 \\
\hline \multirow[t]{4}{*}{ KIDSCREEN-P } & Pre & 73 & $37.22(3.77)$ & Pre-12MFU & $0.48(0.21,0.75)$ & $<0.001$ \\
\hline & Post & 73 & $38.60(4.21)$ & Pre-Post & $0.34(0.14,0.54)$ & 0.008 \\
\hline & $3 \mathrm{MFU}$ & 66 & $38.55(5.17)$ & Post-3MFU & $0.02(-0.25,0.22)$ & 0.863 \\
\hline & $12 \mathrm{MFU}$ & 59 & $36.69(4.94)$ & $3 \mathrm{MFU}-12 \mathrm{MFU}$ & $0.22(-0.01,0.45)$ & 0.034 \\
\hline
\end{tabular}

ICBT internet-delivered cognitive behavioural therapy; CSR Clinician Severity Rating; CGAS Children's Global Assessment Scale; $R C A D S$ - $C$ or $P$ Revised Children's Anxiety and Depression Scale-child and parent versions; WSAS-C or P Work and Social Adjustment Scale-Child and parent versions; KIDSCREEN$\mathrm{C}$ or $\mathrm{P}=\mathrm{KIDSCREEN}-10$-child and parent versions. Note. Mean and standard deviation based on the observed data; effect size (Cohen's $d$ ) and $p$ value based on the estimated means derived from the linear mixed model; RCADS-C/P Anxiety symptoms sub-scale only; the missing data due to either (1) drop-out, (2) parent and/or child forgetting to, or not wanting to, log in on platform to answer questionnaires or (3) Assessor forgetting to log assessment in case report form
Seven participants (10.3\%) in the R-ICBT group received additional treatment elsewhere (i.e., local CAMHS) during the follow-up period: three participants who were on SSRI medication prior to starting ICBT increased their dosage during the follow-up period and four participants were referred to their local CAMHS by a clinician in the trial during the follow-up period. The results remained largely unchanged when the receipt of additional treatments during the follow-up were introduced in the models as a covariate
( $p=0.871$ for the additional treatment by time interaction effect).

\section{Long-term outcomes for non-remitters after ICBT}

Thirty-seven participants were assessed as non-remitters at 3MFU. Eighteen of the non-remitters (48.6\%) accepted the offer to receive additional F2F CBT, of which all completed the treatment. The mean number of face-to-face sessions 
was $M=6.72(S D=2.59$; $\min -\max =2-10)$; supplementary Table 6 lists the perceived reasons why ICBT had not worked for these participants.

Despite not achieving remission status at post-treatment, these patients still improved significantly from pre- to postICBT $(t=3.95, p=0.001 ; d=0.99 ; 95 \% \mathrm{CI}=0.30-1.67)$. However, they improved the most after receiving additional F2F CBT (i.e., between 3 and 6MFU; $t=4.62, p<0.001$ ) with a large effect size $(d=1.53 ; 95 \% \mathrm{CI}=0.55-2.51)$. No change in the symptom severity was observed between posttreatment and $3 \mathrm{MFU}(t=0.77, p=0.446)$. Considering the entire treatment received (ICBT plus F2F CBT), the overall effect size from pre-ICBT to $12 \mathrm{MFU}$ on the CSR was very large $(d=2.27$; 95\% CI $=1.03-3.50)$; see Supplementary Table 7 for a full summary of primary- and secondary outcomes from pre-treatment to $12 \mathrm{MFU}$ for participants in this group.

At 6MFU, directly after having completed F2F CBT, 12 participants (70.6\%) no longer fulfilled their principal anxiety disorder and ten participants $(58.8 \%)$ were free from all their anxiety disorders. At $12 \mathrm{MFU}, 14$ participants (82.4\%) were in remission and ten participants $(58.8 \%)$ were free from all their anxiety disorders. No participants relapsed between 6 and 12MFU.

Nineteen non-remitters after ICBT turned down the offer to receive additional treatment at $3 \mathrm{MFU}$, of whom $36.8 \%$ $(n=7)$ did not want to and did not receive any additional treatment during the entire follow-up. Twelve participants $(63.2 \%)$ turned down the offer to receive additional treatment due to already receiving additional treatment at their local CAMHS. All these participants had been referred to their local CAMHS by a clinician working in the trial. Four

Change on clinician severity rating (CSR) of the principal anxiety disorder

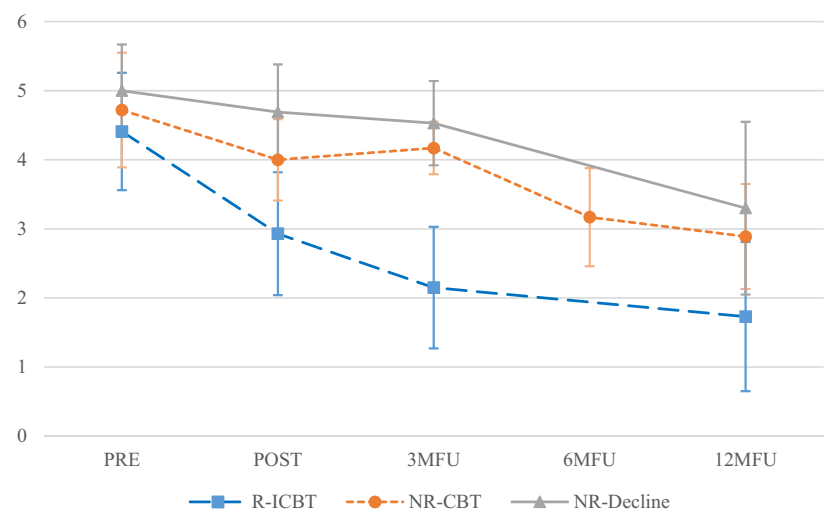

Fig. 2 Change on primary outcome measure, clinician severity rating of the principal anxiety disorder, CSR, (left) as well as clinically assessed functional impairment, CGAS (right) based on whether participants three-months after completed ICBT were in remission (R-ICBT; $n=73$ ), not in remission receiving additional face-to-face participants were referred due to being in need of additional treatment for an anxiety disorder and not being able to wait until 3MFU when additional treatment would be offered as part of the trial. The other eight participants were referred due to another mental health condition other than their principal anxiety disorder. For a full description and overview of referrals to local CAMHS for non-remitters turning down the offer to receive additional F2F CBT due to receiving treatment elsewhere, see Supplemental Fig 2.

Participants in NR-Decline did not improve significantly from pre- to post-treatment $(t=1.47, p=0.148)$ or between post-treatment and 3MFU $(t=0.78, p=0.442)$. However, they did improve significantly between 3 and $12 \mathrm{MFU}$ $(t=5.84, p<0.001)$ with a large effect size $(d=1.12 ; 95 \%$ $\mathrm{CI}=0.48-1.76)$. Considering the whole study period, the overall effect size from pre-ICBT to $12 \mathrm{MFU}$ on the CSR was large $(d=1.51 ; 95 \% \mathrm{CI}=0.69-2.34)$; see Supplementary Table 8 for a full summary of primary and secondary outcomes from pre-treatment to $12 \mathrm{MFU}$ for participants in this group.

Figure 2 shows the change over time for participants in the R-ICBT, NR-CBT and NR-Decline groups on clinician severity rating (CSR) of the principal anxiety disorder as well as clinician-assessed functional impairment (CGAS). At 12MFU, eleven participants (57.9\%) in NR-Decline were assessed as being in remission and ten participants (52.6\%) were free from all anxiety disorders. Figure 3 shows proportions of participants in the R-ICBT, NR-CBT and NRDecline free from their principal anxiety disorder as well as free from all their anxiety disorders at $12 \mathrm{MFU}$. No severe adverse events were reported from either ICBT- or F2F CBT treatment.

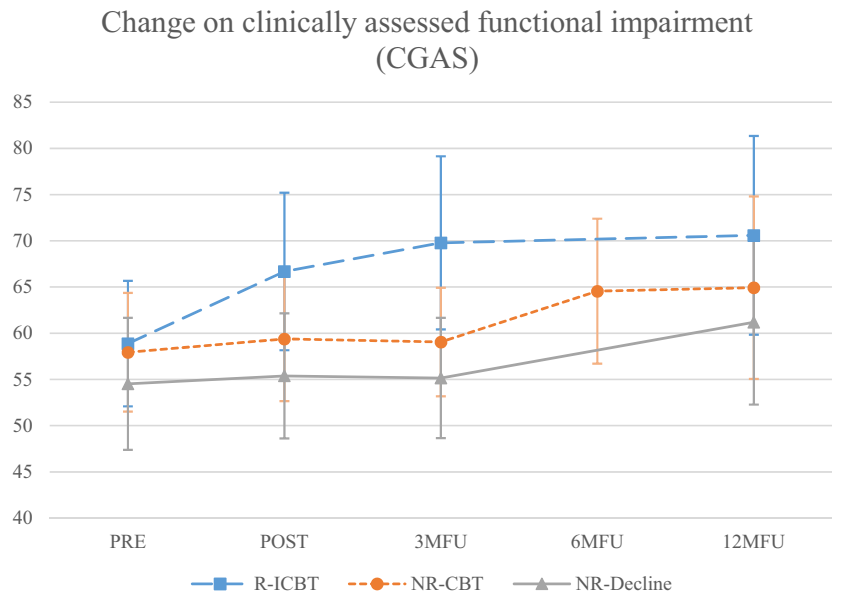

CBT (NR-CBT; $n=18$ ), or not in remission declining the offer to receive additional face-to-face CBT (NR-Decline; $n=19$ ). Assessments points in relation to when ICBT was completed; Six-months follow-up (6MFU) only for participants receiving additional face-toface CBT, i.e., corresponds to the post face-to-face CBT assessment 
Fig. 3 Proportion of participants in remission at $12 \mathrm{MFU}$. $R-I C B T$ participants assessed as remitters at three-months after completed ICBT; $N R-C B T$ participants assessed as not in remission receiving additional face-to-face CBT 3-months after completed ICBT; $N R$ Decline participants not in remission declining the offer to receive additional face-to-face CBT 3-months after completed ICBT

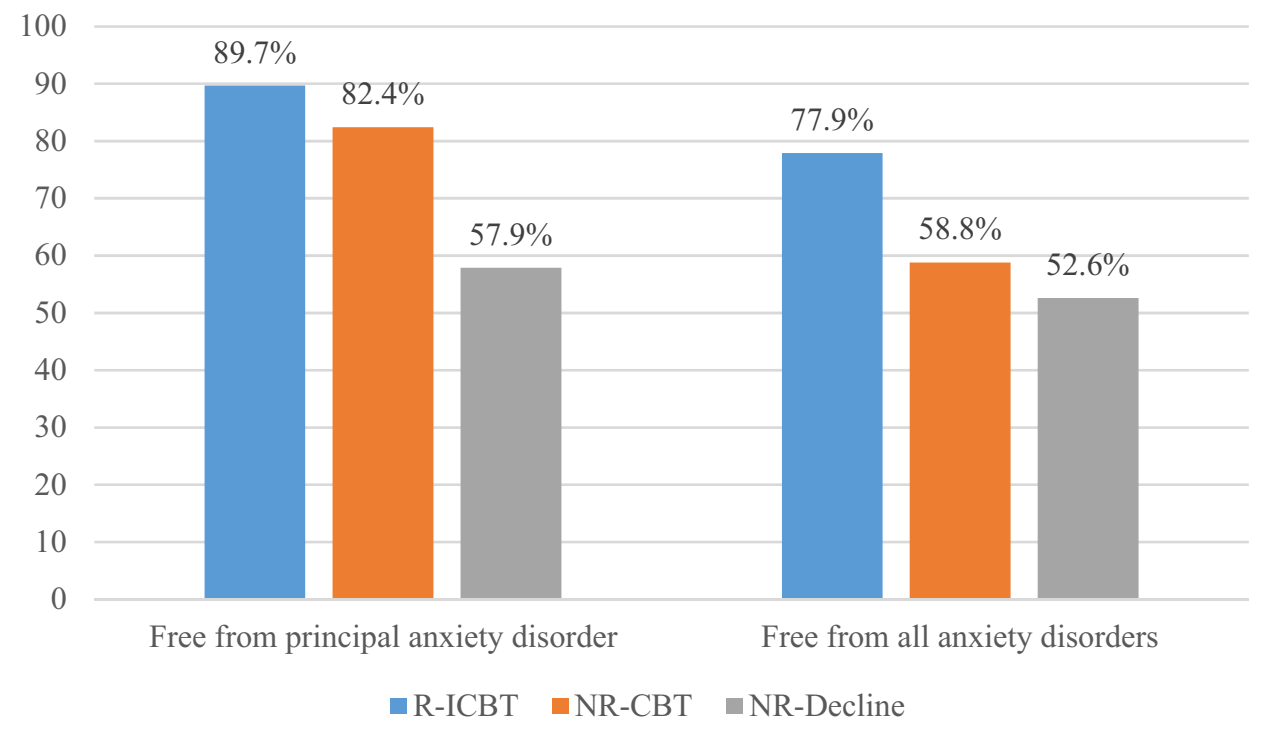

occurred after F2F CBT. By the end of the 12MFU, these patients had improved as much as those who initially responded to ICBT. These results are in line with two other trials naturalistically investigating the effects of additional CBT for children with anxiety disorders who did not respond sufficiently after first receiving parental-guided CBT [15] or attention bias modification training [14].

Somewhat unexpectedly, only 18 participants (48.6\%), out of the 37 non-remitters, accepted the offer of additional F2F CBT at 3MFU. Participants declining the offer to receive additional treatment were more severe at pretreatment, had a higher number of comorbid anxiety disorders and higher rates of participants with social anxiety disorder. Though many of these patients received treatments elsewhere, participants declining additional F2F CBT did not improve as much as participants in the other two groups. As this is a naturalistic study, we are cautious not to attribute these differences to the receipt of additional F2F CBT. Most of the participants declining the offer to receive additional treatment at $3 \mathrm{MFU}$, did so because they had already sought additional treatment elsewhere. This could indicate that, for at least a sub-group of individuals, stepping up treatment should be considered earlier than 3MFU. There is a clear need to conduct further research into the identification of early predictors of treatment response in ICBT to better guide clinical decision making. Intuitively, ICBT may not be the first treatment of choice for patients with severe anxiety disorders or with complex comorbidities. However, this may not necessarily be the case; some participants who had higher level of anxiety and comorbidity at pre-treatment, responded well to ICBT and additional F2F CBT and maintained their gains at follow-up.

Strengths of this study include the large sample size, high participant retention and limited missing data. Additional 
strengths were the systematic recording of, and statistical adjustment for, additional treatments during the follow-up and the implementation of highly structured F2F manual in the context of a stepped-care approach. This study also had some limitations. First, this was a naturalistic follow-up study and, as such, we could not control for the passage of time. This is unfortunately the case for the majority of trials investigating the long-term outcomes of psychological treatments for paediatric anxiety disorders [28]. Thus, some of the observed improvements may be due to other factors such as environmental changes or normal maturation of the children. Second, participants in this study were less severe with regards to the severity of their principal anxiety disorder compared to other trials on ICBT for childhood anxiety disorders (e.g., [8].). Thus, the results may not generalise to the most severe end of the anxiety disorder spectrum. Third, we did not randomise participants into a stepped-care approach or a "gold standard" treatment, which would be the ideal design to test, e.g., hypothesised health economic benefits of a stepped care approach. Notwithstanding, we believe that our study highlights the feasibility of implementing such a model in regular healthcare and encourages further work in this area.

\section{Conclusion}

This study reported on the long-term naturalistic outcomes of participants in a clinical trial of ICBT for paediatric anxiety disorders within a stepped-care model where nonremitters were offered additional face-to-face (F2F) treatment. Patients classified as remitters after ICBT maintained their gains and even improved further 1 year after the end of treatment, even when statistically controlling for supplementary service use throughout the follow-up period. Nonremitters after ICBT who choose to receive additional F2F CBT improved similarly as remitters of ICBT, the largest improvement occurring after F2F CBT. It may be feasible to implement ICBT in regular healthcare adopting a stepped care approach.

Acknowledgements The authors would like to thank the contribution of Martin Persson MSc, Vide Gotby Olsson MSc, Erika Sundqvist MSc, Ann-Sofie Ersson MSc, and Kajsa Mitsell MSc, for assisting with assessment and treatment of participants in this trial, Ulrika Thulin $\mathrm{PhD}$, who helped create the BiP Anxiety programme, Gun Billström for helping with study administration, and the directors of the childand adolescent mental health services in Stockholm for supporting this study.

Author contributions $\mathrm{MJ}, \mathrm{SV}, \mathrm{DM}-\mathrm{C}, \mathrm{BL}, \mathrm{JH}, \mathrm{L}-\mathrm{GÖ}$ and ES contributed to the conception and design of the trial. MJ, SV, DM-C and BL contributed to the data analysis. MJ, SV, TW, MN and JH contributed to the acquisition of data. MJ drafted the work and all other authors contributed to revising it critically for important intellectual content.
All authors approved the final version of the paper to be published and agree to be accountable for all aspects of the work in ensuring that questions related to the accuracy or integrity of any part of the work are appropriately investigated and resolved.

Funding Open access funding provided by Karolinska Institute. The Swedish Research Council for Health, Working Life and Welfare (Forte 2014-4052) and Stockholm County Council (HNSV 14099) funded this trial.

\section{Compliance with ethical standards}

Conflict of interest MJ, SV and ES are the developers of the BiP Anxiety treatment protocol. BL is a Shareholder in DahliaQomit AB. DM-C received personal fees from UpToDate and Elsevier; grants from NIMH, Vetenskapsradet, Jane and Dan Olsson Foundation, Hjarnfonden and NIHR UK. All other authors have declared no competing or potential conflict of interest.

Open Access This article is licensed under a Creative Commons Attribution 4.0 International License, which permits use, sharing, adaptation, distribution and reproduction in any medium or format, as long as you give appropriate credit to the original author(s) and the source, provide a link to the Creative Commons licence, and indicate if changes were made. The images or other third party material in this article are included in the article's Creative Commons licence, unless indicated otherwise in a credit line to the material. If material is not included in the article's Creative Commons licence and your intended use is not permitted by statutory regulation or exceeds the permitted use, you will need to obtain permission directly from the copyright holder. To view a copy of this licence, visit http://creativecommons.org/licenses/by/4.0/.

\section{References}

1. Polanczyk GV, Salum GA, Sugaya LS, Caye A, Rohde LA (2015) Annual Research review: a meta-analysis of the worldwide prevalence of mental disorders in children and adolescents. J Child Psychol and Psych 56(3):345-365. https://doi.org/10.1111/ jcpp. 12381

2. Copeland WE, Angold A, Shanahan L, Costello EJ (2014) Longitudinal patterns of anxiety from childhood to adulthood: the great smoky mountains study. J Am Acad Child Adolesc Psychiatry 53(1):21-33. https://doi.org/10.1016/j.jaac.2013.09.017

3. James AC, James G, Cowdrey FA, Soler A, Choke A (2015) Cognitive behavioural therapy for anxiety disorders in children and adolescents. Coch Database Syst Rev. https://doi. org/10.1002/14651858.CD004690.pub3

4. Kazdin AE (2017) Addressing the treatment gap: a key challenge for extending evidence-based psychosocial interventions. Behav Res Ther 88:7-18. https://doi.org/10.1016/j.brat.2016.06.004

5. Hollis C, Falconer CJ, Martin JL, Whittington C, Stockton S, Glazebrook C, Davies EB (2017) Annual research review: digital health interventions for children and young people with mental health problems-a systematic and meta-review. J Child Psychol Psychiatry 58(4):474-503. https://doi.org/10.1111/jcpp.12663

6. Jolstedt M, Wahlund T, Lenhard F, Ljótsson B, Mataix-Cols D, Nord M, Öst L-G, Högström J, Serlachius E, Vigerland S (2018) Efficacy and cost-effectiveness of therapist-guided internet cognitive behavioural therapy for paediatric anxiety disorders: a singlecentre, single-blind, randomised controlled trial. Lancet Child Adolesc Health 2(11):792-801. https://doi.org/10.1016/S2352 $-4642(18) 30275-X$ 
7. Kazdin AE (2019) Annual research review: expanding mental health services through novel models of intervention delivery. J Child Psychol Psych 60(4):455-472. https://doi.org/10.1111/ jcpp.12937

8. March S, Spence SH, Donovan CL (2009) The efficacy of an internet-based cognitive-behavioral therapy intervention for child anxiety disorders. J Pediatr Psychol 34(5):474-487. https://doi. org/10.1093/jpepsy/jsn099

9. Spence SH, Donovan CL, March S, Gamble A, Anderson RE, Prosser S, Kenardy J (2011) A randomized controlled trial of online versus clinic-based cbt for adolescent anxiety. J Consult Clin Psychol 79(5):629-642. https://doi.org/10.1037/a0024512

10. Vigerland S, Serlachius E, Thulin U, Andersson G, Larsson JO, Ljótsson B (2017) Long-term outcomes and predictors of internet-delivered cognitive behavioral therapy for childhood anxiety disorders. Behav Res Ther 90:67-75. https://doi.org/10.1016/j. brat.2016.12.008

11. Ollendick TH, Öst L-G, Farrell LJ (2018) Innovations in the psychosocial treatment of youth with anxiety disorders: implications for a stepped care approach. Evid-Based Ment Health 21(3):112. https://doi.org/10.1136/eb-2018-102892

12. Bower P, Gilbody S (2005) Stepped care in psychological therapies: access, effectiveness and efficiency: narrative literature review. Br J Psychiatry 186(1):11-17. https://doi.org/10.1192/ bjp.186.1.11

13. Rapee RM, Lyneham HJ, Wuthrich V, Chatterton ML, Hudson JL, Kangas M, Mihalopoulos C (2017) Comparison of stepped care delivery against a single, empirically validated cognitive-behavioral therapy program for youth with anxiety: a randomized clinical trial. J Am Acad Child Adolesc Psychiatry 56(10):841-848. https ://doi.org/10.1016/j.jaac.2017.08.001

14. Pettit JW, Rey Y, Bechor M, Melendez R, Vaclavik D, Buitron V, Bar-Haim Y, Pine DS, Silverman WK (2017) Can less be more? Open trial of a stepped care approach for child and adolescent anxiety disorders. J Anxiety Disord 51:7-13. https://doi. org/10.1016/j.janxdis.2017.08.004

15. van der Leeden AJM, van Widenfelt BM, van der Leeden R, Liber JM, Utens EM, Treffers PD (2011) Stepped care cognitive behavioural therapy for children with anxiety disorders: a new treatment approach. Behav Cogn Psychother 39(1):55-75. https://doi. org/10.1017/S1352465810000500

16. Silverman WK, Albano AM (1996) Anxiety disorders interview schedule for DSM-IV: parent interview schedule. Greywind Publications, Albany, NY

17. Silverman WK, Saavedra LM, Pina AA (2001) Test-retest reliability of anxiety symptoms and diagnoses with the anxiety disorders interview schedule for DSM-IV: child and parent versions. J Am Acad Child Adolesc Psychiatry 40(8):937-944. https://doi. org/10.1097/00004583-200108000-00016
18. Wood JJ, Piacentini JC, Bergman RL, McCracken J, Barrios V (2002) Concurrent validity of the anxiety disorders section of the anxiety disorders interview schedule for DSM-IV: child and parent versions. J Clin Child Adolesc 31(3):335-342. https://doi. org/10.1207/S15374424JCCP3103_05

19. Shaffer D, Gould MS, Brasic J, Ambrosini P, Fisher P, Bird H, Aluwahlia S (1983) A children's global assessment scale (CGAS). Arch Gen Psychiatry 40(11):1228-1231. https://doi.org/10.1001/ archpsyc.1983.01790100074010

20. Chorpita BF, Yim L, Moffitt C, Umemoto LA, Francis SE (2000) Assessment of symptoms of DSM-IV anxiety and depression in children: a revised child anxiety and depression scale. Behav Res Ther 38(8):835-855. https://doi.org/10.1016/S0005 -7967(99)00130-8

21. Spence SH (1998) A measure of anxiety symptoms among children. Behav Res Ther 36(5):545-566. https://doi.org/10.1016/ S0005-7967(98)00034-5

22. Jassi A, Lenhard F, Krebs G, Gumpert M, Jolstedt M, Andrén P, Mataix-Cols D (2019) The work and social adjustment scale, youth and parent versions: psychometric evaluation of a brief measure of functional impairment in young people. Child Psyc Hum Dev 1:4. https://doi.org/10.31234/osf.io/f8zev

23. Ravens-Sieberer U, Erhart M, Rajmil L, Herdman M, Auquier P, Bruil J, Power M, Duer W, Abel T, Czemy L (2010) Reliability, construct and criterion validity of the KIDSCREEN-10 score: a short measure for children and adolescents' well-being and healthrelated quality of life. Qual Life Res 19(10):1487-1500. https:// doi.org/10.1007/s11136-010-9706-5

24. Zigmond AS, Snaith RP (1983) The hospital anxiety and depression scale. Acta Psychiatr Scand 67(6):361-370. https://doi. org/10.1111/j.1600-0447.1983.tb09716.x

25. Hesser H (2015) Modeling individual differences in randomized experiments using growth models: recommendations for design, statistical analysis and reporting of results of internet interventions. Internet Interv 2(2):110-120. https://doi.org/10.1016/j.inven t.2015.02.003

26. Feingold A (2009) Effect sizes for growth-modeling analysis for controlled clinical trials in the same metric as for classical analysis. Psychol Methods 14(1):43. https://doi.org/10.1037/a0014699

27. Piacentini J, Bennett S, Compton SN, Kendall PC, Birmaher B, Albano AM, March J, Sherrill J, Sakolsky D, Ginsburg G (2014) 24-and 36-week outcomes for the child/adolescent anxiety multimodal study (CAMS). J Am Acad Child Adolesc Psychiatry 53(3):297-310. https://doi.org/10.1016/j.jaac.2013.11.010

28. Gibby BA, Casline EP, Ginsburg GS (2017) Long-term outcomes of youth treated for an anxiety disorder: a critical review. Clin Child Fam Psych 20(2):201-225. https://doi.org/10.1007/s1056 7-017-0222-9 